\title{
Transmission of infection to liver transplant recipients from donors with infective endocarditis: lessons learned
}

\author{
M.H. Miceli, M. Gonulalan, M.B. Perri, L. Samuel, M.A. Al Fares, K. \\ Brown, D.A. Bruno, M. Zervos, M. Ramesh, G. Alangaden. Trans- \\ mission of infection to liver transplant recipients from donors with \\ infective endocarditis: lessons learned. \\ Transpl Infect Dis 2015: 17: 140-146. All rights reserved \\ Abstract: Donors not meeting standard criteria, such as those with \\ bacteremia, are now being used in response to the increasing need \\ for organs for transplantation. Recommended strategies to prevent \\ the occurrence of donor-derived bacteremia include the use of \\ directed antibiotic prophylaxis. However, this approach does not \\ eliminate the risk of infection transmission. Similarly, the \\ management of organ recipients from donors with infective \\ endocarditis (IE) remains uncharacterized. We report 2 cases of \\ donor-derived bacterial infections in liver transplant recipients \\ despite pathogen-specific antibiotic prophylaxis. In both instances, \\ the donors had documented IE treated with appropriate \\ antimicrobial therapy and clearance of bacteremia. Recipients had \\ very distinctive clinical outcomes likely related to pathogen \\ virulence and the extent of donor infection. Persistent infection in \\ the transplanted liver should be suspected in organ recipients of a \\ liver from donors with IE, despite the absence of bacteremia at the \\ time of death and organ procurement. For eradication, recipients \\ may require prolonged pathogen-directed antimicrobial therapy, \\ such as is used for endovascular infections. Prompt recognition of \\ donors with IE, appropriate notification, and prolonged antibiotic \\ prophylaxis are key to reducing the risk of such donor-derived \\ infections.
}

\author{
M.H. Miceli', M. Gonulalan ${ }^{2}$, M.B. \\ Perri $^{2}$, L. Samuel ${ }^{3}$, M.A. Al Fares ${ }^{5}$, \\ K. Brown ${ }^{4}$, D.A. Bruno ${ }^{4}$, M. Zervos ${ }^{2}$, \\ M. Ramesh', G. Alangaden ${ }^{2}$ \\ ${ }^{1}$ Department of Internal Medicine, Division of Infectious \\ Diseases, University of Michigan School of Medicine, Ann \\ Arbor, Michigan, USA, ${ }^{2}$ Department of Internal Medicine, \\ Division of Infectious Diseases, Henry Ford Health \\ System, Detroit, Michigan, USA, ${ }^{3}$ Department of \\ Pathology, Henry Ford Health System, Detroit, Michigan, \\ USA, ${ }^{4}$ Transplant Institute, Henry Ford Health System, \\ Detroit, Michigan, USA, ${ }^{5}$ Department of Internal \\ Medicine, Saint John Providence Health System, Detroit, \\ Michigan, USA
}

Key words: infective endocarditis; liver transplant; donor transmission

\author{
Correspondence to: \\ Marisa H. Miceli, Department of Internal Medicine, \\ Division of Infectious Diseases, University of \\ Michigan Medical School, 3120 Taubman Center, \\ 1500 E. Medical Center Dr. SPC 5378, \\ Ann Arbor, Ml 48109-5378, USA \\ Tel: (734) 647-5899 \\ Fax: (734) 615-2737 \\ E-mail: mmiceli@med.umich.edu
}

Received 11 March 2014, revised 25 July 2014, 26

September 2014, accepted for publication 7

October 2014

DOI: 10.1111/tid.12330

Transpl Infect Dis 2015: 17: 140-146
Organs from donors not meeting standard donor criteria, such as donors with bacteremia or infective endocarditis (IE), are now utilized in response to the increasing need for organs for transplantation (1). Recommended strategies to prevent the occurrence of donor-derived bacteremia include the use of directed antibiotic prophylaxis (2). However, this approach does not eliminate the risk of infection transmission. Similarly, the management of organ recipients from donors with IE remains uncharacterized. Between December 2012 and March 2013, 2 cases of donorderived bacterial infections in liver transplant (LT) recipients were identified at Henry Ford Hospital, an 802-bed tertiary care center in Detroit. In both instances 
the donors had been appropriately treated for IE and had clearance of bacteremia at the time of death.

\section{Case reports}

\section{Case 1}

A 59-year-old man, with congenital aortic stenosis status post aortic valve replacement, and end-stageliver disease secondary to hepatitis $\mathrm{C}$ complicated with hepatocellular carcinoma treated with chemoembolization and radio frequency ablation, received an LT. Immunosuppressive regimen included induction with basiliximab and maintenance with tacrolimus, mycophenolate mofetil, and prednisone. Opportunistic infections prophylaxis included fluconazole, trimethoprimsulfamethoxazole, and valganciclovir as per protocol.

The donor was a 31-year-old woman with lupus erythematous and end-stage renal disease on hemodialysis performed through a PermCath, who developed fever during hemodialysis. Blood cultures the day before and for 5 subsequent days following hospital admission were positive for vancomycin- and ampicillinsusceptible Enterococcus faecalis (VSE). Transthoracic echocardiogram showed a mobile vegetation on the posterior mitral valve leaflet $(0.85 \mathrm{~cm} \times 0.5 \mathrm{~cm})$ and mild mitral valve regurgitation. The patient (donor) was treated for IE with intravenous (IV) vancomycin and subsequently with high-dose ampicillin (VSE demonstrated high-level resistance to aminoglycoside). Clinical course of the donor was further complicated by poor venous access after removal of the PermCath. The patient subsequently had a new PermCath placed after clearance of bacteremia on hospital day 10 . She then developed acute deep vein thrombosis of the right lower extremity requiring anticoagulation.

On day 17 , the patient sustained a fall with blunt head trauma. Computerized tomography (CT) scan of the head showed a large hemorrhage in the right basal ganglia with mid-line shift. The patient had progressive neurologic status deterioration and subsequently needed resuscitation for cardiac arrest with pulseless electrical activity. After resuscitation, the patient remained unresponsive with asymmetric pupils. The patient was subsequently declared brain dead. Only the liver was accepted for transplantation. Of note, blood cultures from days 6,10 , and 17 of hospitalization, and at time of organ procurement, were negative. The donor did not undergo an autopsy.

The LT recipient received cefepime and metronidazole for surgical prophylaxis. On post-transplant day 1 , accessory left hepatic artery bleeding developed, necessitating an exploratory laparotomy and ligation of the artery. Because of postoperative fevers and leukocytosis, cefepime and metronidazole were continued and vancomycin was added on postoperative day (POD) 1. Blood cultures obtained on POD 1 were negative. On POD 5, Infectious Diseases was consulted because of persistence of fevers despite broad-spectrum antibiotic coverage. Given the known history of Enterococcus species bacteremia in the donor, vancomycin was switched to daptomcyin $(8 \mathrm{mg} / \mathrm{kg} /$ day $)$ pending detailed information about the donor's infection from Gift of Life (GOL). Within the next day, GOL confirmed recent history of persistent bacteremia with VSE sensitive to ampicillin, vancomycin, and daptomycin, and high-level gentamicin synergy resistance. Based on this information, IV ampicillin ( $1 \mathrm{~g}$ q $6 \mathrm{~h}$ ) was added. The patient remained febrile and on POD 9 developed hypotension.

A CT scan of the abdomen showed a large intraabdominal hematoma. A second laparotomy and evacuation of the hematoma was performed. Cultures of the hematoma yielded E. faecalis. Ampicillin was discontinued owing to development of a drug-related rash. The patient completed an additional 3 weeks of therapy with daptomycin. On work-up, no other site of infection including IE was identified.

The patient remains well at 6 months of follow up. The antibiograms (Table 1) and pulse field gel electrophoresis (PFGE) typing patterns (Fig. 1) of the VSE isolated from the donor blood cultures and the recipient hematoma cultures were identical.

\section{Case 2}

A 41-year-old man, with a model for end-stage-liver disease (MELD) score of 40 secondary to primary sclerosing cholangitis and ulcerative colitis, received an LT. Immunosuppression included induction with antithymocyte globulin followed by maintenance with tacrolimus, mycophenolate mofetil, and prednisone. At the time of LT the recipient was on IV daptomycin (4 mg/ $/ \mathrm{kg} /$ day) for the treatment of soft tissue infection. He had completed treatment for an episode of Clostridium difficile-associated diarrhea (CDAD). Surgical prophylaxis in the recipient included cefepime and metronidazole, in addition to the daptomycin therapy for cellulitis. Opportunistic infections prophylaxis included fluconazole, trimethoprim-sulfamethoxazole, and valganciclovir, as per protocol.

The donor was a 22-year-old man, an active IV drug user who was found unresponsive in the street. He was taken to the local Emergency Department, where he 
Antibiotic susceptibilities of the bacterial isolates

\begin{tabular}{|c|c|c|c|c|c|c|c|}
\hline \multirow[b]{2}{*}{ Cases } & \multirow[b]{2}{*}{ Pathogen } & \multicolumn{6}{|c|}{ Antibiotic susceptibilities (MIC $\mu \mathrm{g} / \mathrm{mL}$ ) } \\
\hline & & AMP & OXA & VAN & GEN & $\mathrm{DAP}^{*}$ & Other \\
\hline \multicolumn{8}{|l|}{ Case 1} \\
\hline Donor & $\begin{array}{l}\text { Enterococcus } \\
\text { faecalis }\end{array}$ & $\mathrm{S}(\mathrm{MIC}<2)$ & NA & $\mathrm{S}(\mathrm{MIC}=1)$ & $\mathrm{R}$ & NA & $\mathrm{R}$ \\
\hline Recipient & E. faecalis & $\mathrm{S}(\mathrm{MIC}<2)$ & NA & $\mathrm{S}(\mathrm{MIC}=1)$ & $\mathrm{R}$ & NA & $\mathrm{R}$ \\
\hline \multicolumn{8}{|l|}{ Case 2} \\
\hline Donor & MRSA & NA & $\mathrm{R}$ & $\mathrm{S}(\mathrm{MIC} \leq 2)$ & $\mathrm{S}(\mathrm{MIC} \leq 0.5)$ & NA & $\begin{array}{r}\text { Clindamycin } \\
(\mathrm{MIC} \leq 0.5)\end{array}$ \\
\hline $\begin{array}{l}\text { Recipient (First episode, } \\
6 \text { Dec 2012) }\end{array}$ & MRSA & NA & $\mathrm{R}(\mathrm{MIC}>4)$ & $\mathrm{S}(\mathrm{MIC}=1.5)$ & $\mathrm{S}(\mathrm{MIC} \leq 0.5)$ & $\mathrm{S}(\mathrm{MIC}=1)$ & $\begin{array}{l}\text { Clindamycin - } \\
\mathrm{S}(\mathrm{MIC} \leq 0.25)\end{array}$ \\
\hline $\begin{array}{l}\text { Recipient (Second episode, } \\
16 \text { Feb 2013) }\end{array}$ & MRSA & NA & $\mathrm{R}(\mathrm{MIC}>4)$ & $S(M I C=2)$ & $S(M I C \leq 0.5)$ & $\mathrm{S}(\mathrm{MIC} \leq 0.25)$ & $\begin{array}{l}\text { Clindamycin - } \\
\mathrm{S}(\mathrm{MIC} \leq 0.25)\end{array}$ \\
\hline $\begin{array}{l}\text { Recipient (Third episode, } \\
5 \text { May 2013) }\end{array}$ & MRSA $(5 / 5 / 13)$ & NA & $\mathrm{R}(\mathrm{MIC}>4)$ & $S(M I C=1.5)$ & $\mathrm{S}(\mathrm{MIC}<0.5)$ & $S(M I C=1)$ & $\begin{array}{l}\text { Linezolid - } \\
\qquad \mathrm{S}(\mathrm{MIC}=2)\end{array}$ \\
\hline
\end{tabular}

${ }^{*}$ E-test was used for determining the MIC for daptomycin.

MIC, minimum inhibitory concentration; AMP, ampicillin; OXA, oxacillin; VAN, vancomycin; GEN, gentamicin; DAP, daptomycin; S, sensitive; NA, not available; R, resistant; MRSA, methicillin-resistant Staphylococcus aureus.

Table 1

was found to have an elevated temperature of $105^{\circ} \mathrm{F}$ and a large occipital bleed. Broad-spectrum antibiotics (i.e., vancomycin and piperacillin-tazobactam) were started immediately. Initial blood cultures were reported positive for gram-positive cocci in clusters, and the patient was continued on vancomycin and piperacillin-tazobactam. A transthoracic echocardiogram showed a mobile mass on anterior mitral valve leaflet with moderate-to-severe mitral regurgitation, indicative of left-sided IE. His blood cultures were confirmed positive for methicillin-resistant Staphylococcus aureus (MRSA). Subsequent blood cultures were negative, documenting clearance of bacteremia. Despite aggressive supportive measures, he remained unresponsive, and was declared brain dead. By the time of organ procurement, the donor had received antimicrobial therapy active against MRSA and had remained afebrile for more than 2 days. Blood cultures at the time of procurement showed no growth. The liver, both lungs, one kidney and pancreas, and the second kidney were transplanted to 4 recipients. No evidence of intraabdominal infection was noted at the time of organ procurement. Unfortunately, the donor did not undergo an autopsy.

Intraoperative donor liver biopsy at the time of transplant was culture negative, but blood cultures, obtained from the recipient $3 \mathrm{~h}$ after the LT procedure, yielded MRSA. Daptomycin dose was increased to
$8 \mathrm{mg} / \mathrm{kg} /$ day on POD 1 after transplant surgery, once the donor MRSA susceptibilities became available. Repeat blood cultures obtained 5 days after LT were negative. Work-up including ultrasound of the liver and CT scans of the abdomen and pelvis were negative for any deep-seated infection, and multiple repeat blood cultures remained negative.

After documenting clearance of MRSA bloodstream infection (BSI), and negative work-up for deep-seated MRSA infection, a peripherally inserted central catheter (PICC) line was placed for long-term IV antibiotic treatment. The patient remained asymptomatic and clinically stable, and was discharged to a rehabilitation center where he completed 3 weeks of therapy with high-dose daptomycin $(8 \mathrm{mg} / \mathrm{kg} /$ day $)$. The PICC line was removed at the end of IV antibiotic treatment.

Seven weeks after discontinuation of daptomycin (week 10 post LT), the patient was hospitalized with fevers and chills, neutropenia, and elevated liver transaminases. Blood cultures were obtained, and empiric therapy with IV vancomycin, cefepime, and metronidazole was initiated. Blood culture yielded MRSA with a vancomycin minimum inhibitory concentration (MIC) of $2 \mathrm{mcg} / \mathrm{mL}$, and the antibiotic regimen was de-escalated to IV vancomycin (goal trough 15$20 \mathrm{mcg} / \mathrm{mL}$ ).

CT chest scan revealed multiple bilateral pulmonary nodular infiltrates and CT scans of the abdomen and 


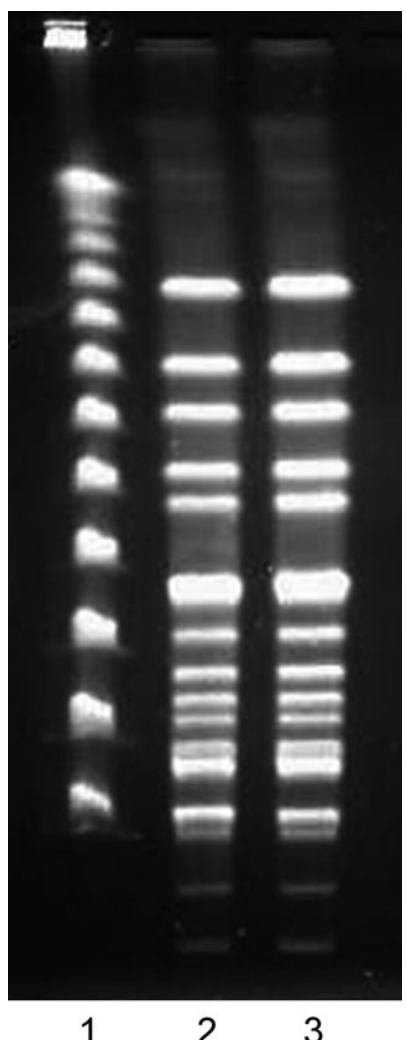

Fig. 1. Pulse field gel electrophoresis patterns of Case 1. Lane 1: Lambda DNA ladder; Lane 2: VSE isolate from recipient; Lane 3: VSE isolate from donor.

pelvis showed splenic infarcts. Blood cultures became negative within $24 \mathrm{~h}$ after therapy. A transthoracic echocardiogram showed no evidence of IE. However, given the concern for septic emboli in the lungs and spleen complicating MRSA bacteremia, the patient received 8 weeks of IV vancomycin therapy.

Two weeks after completion of vancomycin therapy (week 20 post LT) the patient was hospitalized for treatment of recurrent CDAD. During hospitalization, the patient developed fevers and chills. Blood cultures were positive for MRSA, and daptomycin $(8 \mathrm{mg} / \mathrm{kg}$ / day) was restarted. Repeat blood cultures were negative after $48 \mathrm{~h}$ of treatment. An echocardiogram showed no features of IE. The patient completed 8 weeks of therapy with daptomycin.

The patient remained well 3 months after completion of therapy. The MRSA blood culture isolates from the 3 episodes of bacteremia in the recipient had identical PFGE patterns (Fig. 2); antibiotic susceptibilities of each isolate are summarized in Table 1. During an investigation carried out by the Centers for Disease Control and Prevention, MRSA DNA extracted from

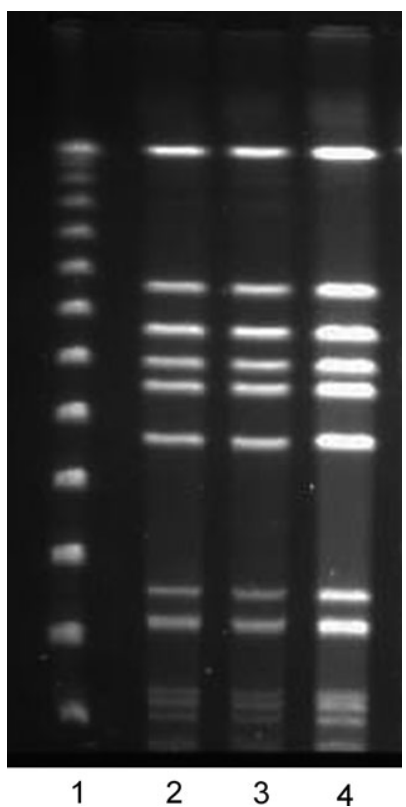

Fig. 2. Pulse field gel electrophoresis patterns of Case 2. Lane 1: Lambda DNA ladder; Lane 2: MRSA isolate from first episode of bacteremia in recipient (6 Dec 2012); Lane 3: MRSA isolate from second episode of bacteremia in recipient (15 Feb 2013); and Lane 4: MRSA from third episode of bacteremia in recipient (5 May 2013).

fixed donor heart valve tissue was matched to the MRSA isolated from the first episode of bacteremia in the recipient, confirming donor-derived transmission (3).

Of note, in addition to our liver recipient, the lung recipient from this donor also developed post-transplant donor-derived MRSA bacteremia, despite vancomycin therapy initiated at the time of transplantation (3). The recipients of the right kidney, and the left kidney and pancreas did not develop MRSA bacteremia. Both these patients received vancomycin prophylaxis for 5 days after transplant surgery because of the donor MRSA bacteremia.

\section{Discussion}

Donor-derived infections among solid organ transplant recipients continue to occur despite the increasing efforts focused on prevention and donor screening (1, 4). The utilization of organs from donors not meeting standard donor criteria has been associated with increased transmission of infections $(5,6)$.

Factors associated with increased risk of infection in the donor include prolonged stay in the intensive care unit ( $>3$ days), whereas older age of the donor was associated with higher rates of infection transmission (5). 
Previously, the presence of bacteremia and IE was considered a contraindication for organ retrieval $(7,8)$. More recent data suggest that organs from a donor with bacteremia and IE can be safely used, provided the donor received appropriate antimicrobial treatment for at least $48 \mathrm{~h}$ and antibiotic therapy is continued in the organ recipient $(5,9-13)$.

We present 2 cases of donor-derived bacterial infections in LT recipients despite pathogen-specific antibiotic coverage. In both instances, the donors had documented left-sided IE treated with pathogen-specific antimicrobial therapy and subsequent clearance of bacteremia. Interestingly, each recipient had a very distinctive clinical course. Our first patient (Case 1) had a less complicated course, having received broadspectrum antibiotic treatment after LT because of signs of infection and the history of donor enterococcal IE. All blood cultures remained negative, probably because of ongoing antimicrobial therapy. However, intra-abdominal hematoma culture was positive for VSE. The patient completed 3 weeks of antibiotics and remains well at 9 months of follow up.

The second recipient (Case 2) had a more complicated clinical course with surveillance blood culture, performed immediately after transplant surgery, being positive for MRSA (first episode), despite being on daptomycin therapy for cellulitis at a dose of $4 \mathrm{mg} / \mathrm{kg} /$ day, compared to the higher dose recommended for the treatment of MRSA BSI (i.e., $6 \mathrm{mg} / \mathrm{kg} /$ day). Repeat blood cultures remained negative after antimicrobial therapy. The patient relapsed (second episode) with MRSA bacteremia, 2 months after completing a 3-week course of antibiotics. Despite completing an additional 8 weeks of directed antibiotic therapy, the patient had a second relapse (the third episode) of MRSA bacteremia.

The first recurrence of MRSA bacteremia in this patient may be the consequence of a shorter duration of therapy, 3 weeks instead of the 4 weeks that current guidelines recommend for the treatment of complicated MRSA BSI (14). The second recurrence, after 8 weeks of vancomycin therapy, may have been in part caused by the higher but still susceptible vancomycin MIC $(2 \mathrm{mcg} / \mathrm{mL}$ ) of the MRSA isolate. Suboptimal response to vancomycin therapy has been reported in infections caused by vancomycin-susceptible strains of MRSA with MIC $>1.5 \mathrm{mcg} / \mathrm{mL}$, as well as a possible upward "creep" in vancomycin MIC (15).

Solid organ transplant recipients at risk for donorderived bacteremia may require prolonged courses of therapy with extremely active antimicrobial treatment, to prevent transmission of infection from donors with IE and to optimize outcomes. In the setting of MRSA IE in the donor, combination therapy with beta-lactams or cotrimoxazole may be an option to improve the antibacterial activity of daptomycin for the prevention and treatment of persistent or relapsing MRSA bacteremia (16-18).

The different clinical outcome of these 2 patients could be explained by the extent of dissemination of infection in the donor and the virulence of the pathogen causing IE. Our first patient, with an intra-abdominal VSE-infected hematoma, had a more benign course. E. faecalis is considered a moderately virulent pathogen and enterococcal IE is rarely associated with metastatic infection. These 2 factors, and the prompt evacuation of the infected hematoma, most likely explain the rapid resolution of the infection in this case. In the second case, the donor had MRSA IE with multiple septic emboli (including to the brain) (3).

Indeed, disseminated foci of infection in bacteremic donors have been associated with a higher risk of transmission (19). Moreover, highly virulent pathogens, such as MRSA, have been associated with posttransplant endovascular infections including mycotic aneurysms (20-23). Donor transmission of bacteremia is reported to occur in up to $5.8 \%$ of LT recipients, despite antibiotic prophylaxis. Previous observations suggest that the risk of transmission is higher among LT recipients compared to other organ recipients $(5,6)$. Doucette et al. (6) have proposed that increased risk of transmission in LT recipients may be multifactorial. It may be attributed to (a) the greater likelihood of infection in the liver, owing to the large mass of tissue and rich vasculature, as well as bacterial retention in the liver secondary to impaired phagocytosis and killing, in the setting of brain death and organ procurement; and (b) because of recipient-related factors, such as a high MELD score, leukopenia, immunosuppression, and poor antibiotic penetration to the transplanted liver (6). In the antibiotic era, septic emboli to the liver and mycotic aneurysms of the hepatic artery are considered rare complications of leftsided IE $(24,25)$. However, these could have been possible factors for the multiple relapses of MRSA infection in our second recipient.

Many questions remain unanswered about the management of recipients of organs from donors with IE, including the optimal duration of antibiotic prophylaxis, and requirement for and timing of surveillance blood cultures (e.g., should all recipients be monitored, or only those receiving organs from donors infected with more virulent pathogens such as MRSA, Pseudomonas aeruginosa, multidrug-resistant organisms). No standard recommendation is available about the duration of antibiotic treatment for bacteremic recipients in this setting. Based on published case series, most experts 
recommend at least 2 weeks of directed antimicrobial therapy $(5,6,9,10)$ and longer treatment $(2-4$ weeks) for $S$. aureus and $P$. aeruginosa infections (19).

To decrease the risk of transmission of infection from liver donors with IE we suggest: (a) detailed review of the clinical data of bacteremic donors to identify possible cases of IE; (b) detailed documentation of the pathogen causing bacteremia, antibiotic susceptibilities, agent used, and duration of antibiotic therapy, response to therapy, and clearance of bacteremia; (c) prompt reporting of this information, and the likelihood of IE, to the transplant teams; and (d) pathogendirected prolonged (3-4 weeks) antibiotic therapy for any LT recipient of a liver from a donor with IE.

Our report illustrates that transmission of infection from donors with IE may occur despite antibiotic prophylaxis. Maximizing effective team communication and detailed donor information are critical to guide the management of these serious infections.

\section{Acknowledgements:}

Author contributions: M.H.M.: Concept/design, data collection/analysis/interpretation, drafting article, critical revision of article, and approval of article. M.G.: Data collection/analysis/interpretation, drafting article, and approval of article. M.B.P.: Molecular studies, sample collection/analysis/interpretation, and approval of article. L.S.: Specimen collection, cultures and result analysis and interpretation, revision of article, and approval of article. M.A.A.: Data collection and interpretation, revision of article, and approval of article. K.B.: Concept, revision of article, approval of article, and other. D.A.B.: Concept, revision of article, approval of article, and other. M.Z.: Concept/design, molecular studies, sample collection/analysis/interpretation, revision of article, and approval of article. M.R.: Concept/ design, data collection, data analysis/interpretation, critical revision of article, and approval of article. G.A.: Concept/design, data collection, data analysis/interpretation, drafting article, critical revision of article, and approval of article.

Presentation: This work was presented as a poster during ID Week 2013, San Francisco, CA, 2013.

\section{References}

1. Wolfe RA, Roys EC, Merion RM. Trends in organ donation and transplantation in the United States, 1999-2008. Am J Transplant 2010; 10: 961-972.
2. Ison MG, Grossi P, AST Infectious Diseases Community of Practice. Donor-derived infections in solid organ transplantation. Am J Transplant 2013; 13 (Suppl 4): 22-30.

3. Wendt JA, Akselrod H, Cohle S, Kaul D, et al. Transmission of methicillin-resistant Staphylococcus aureus infection through solid organ transplantation. Oral Abstracts: Infection in Transplantation and Immunocompromised. ID Week Oct 4, 2013. San Francisco, CA, Abstract \#626

4. Greenwald MA, Kuehnert MJ, Fishman JA. Infectious disease transmission during organ and tissue transplantation. Emerg Infect Dis 2012; 18: e1.

5. Cerutti E, Stratta C, Romagnoli R, et al. Bacterial- and fungalpositive cultures in organ donors: clinical impact in liver transplantation. Liver Transpl 2006; 12: 1253-1259.

6. Doucette KE, Al-Saif M, Kneteman N, et al. Donor-derived bacteremia in liver transplant recipients despite antibiotic prophylaxis. Am J Transplant 2013; 13: 1080-1083.

7. Gore SM, Hinds CJ, Rutherford AJ. Organ donation from intensive care units in England. BMJ 1989; 299: 1193-1197.

8. Nathan HM, Jarrell BE, Broznik B, et al. Estimation and characterization of the potential renal organ donor pool in Pennsylvania. Report of the Pennsylvania Statewide Donor Study. Transplantation 1991; 51: 142-149.

9. Caballero F, Lopez-Navidad A, Domingo P, et al. Successful transplantation of organs retrieved from a donor with enterococcal endocarditis. Transpl Int 1998; 11: 387-389.

10. Caballero F, Lopez-Navidad A, Perea M, et al. Successful liver and kidney transplantation from cadaveric donors with left-sided bacterial endocarditis. Am J Transplant 2005; 5: 781-787.

11. Freeman RB, Giatras I, Falagas ME, et al. Outcome of transplantation of organs procured from bacteremic donors. Transplantation 1999; 68: 1107-1111.

12. Goldberg E, Bishara J, Lev S, et al. Organ transplantation from a donor colonized with a multidrug-resistant organism: a case report. Transpl Infect Dis 2012; 14: 296-299.

13. Gonzalez-Segura C, Pascual M, Garcia Huete L, et al. Donors with positive blood culture: could they transmit infections to the recipients? Transplant Proc 2005; 37: 3664-3666.

14. Liu C, Bayer A, Cosgrove SE, et al. Clinical practice guidelines by the Infectious Diseases Society of America for the treatment of methicillin-resistant Staphylococcus aureus infections in adults and children: executive summary. Clin Infect Dis 2011; 52: 285-292.

15. van Hal SJ, Fowler VG Jr. Is it time to replace vancomycin in the treatment of methicillin-resistant Staphylococcus aureus infections? Clin Infect Dis 2013; 56: 1779-1788.

16. Steed ME, Werth BJ, Ireland CE, et al. Evaluation of the novel combination of high-dose daptomycin plus trimethoprimsulfamethoxazole against daptomycin-nonsusceptible methicillinresistant Staphylococcus aureus using an in vitro pharmacokinetic/pharmacodynamic model of simulated endocardial vegetations. Antimicrob Agents Chemother 2012; 56 : 5709-5714.

17. Di Carlo P, D’Alessandro N, Guadagnino G, et al. High dose of trimethoprim-sulfamethoxazole and daptomycin as a therapeutic option for MRSA endocarditis with large vegetation complicated by embolic stroke: a case report and literature review. Infez Med 2013; 21: 45-49.

18. Dhand A, Bayer AS, Pogliano J, et al. Use of antistaphylococcal beta-lactams to increase daptomycin activity in eradicating persistent bacteremia due to methicillin-resistant Staphylococcus aureus: role of enhanced daptomycin binding. Clin Infect Dis 2011; 53: 158-163. 
19. Echenique IA, Ison MG. Update on donor-derived infections in liver transplantation. Liver Transpl 2013; 19: 575-585.

20. Paredes D, Gambra MP, Cervera C, et al. Characterization of the organ donor with bacteremia. Transplant Proc 2007; 39: 2083-2085.

21. Alamo JM, Gomez MA, Tamayo MJ, et al. Mycotic pseudoaneurysms after liver transplantation. Transplant Proc 2005; 37: 1512-1514.

22. Feltis BA, Lee DA, Beilman GJ. Mycotic aneurysm of the descending thoracic aorta caused by Pseudomonas aeruginosa in a solid organ transplant recipient: case report and review. Surg Infect (Larchmt) 2002; 3: 29-33.

23. Jones VS, Chennapragada MS, Lord DJ, et al. Post-liver transplant mycotic aneurysm of the hepatic artery. J Pediatr Surg 2008; 43: 555-558.

24. Misawa S, Sakano Y, Muraoka A, et al. Septic embolic occlusion of the superior mesenteric artery induced by mitral valve endocarditis. Ann Thorac Cardiovasc Surg 2011; 17: 415-417.

25. Wang $\mathrm{CC}$, Lee $\mathrm{CH}$, Chan $\mathrm{CY}$, et al. Splenic infarction and abscess complicating infective endocarditis. Am J Emerg Med 1021; 2009 (27): e1023-e1025. 